CERN-TH-7076/93

ENSLAPP-A-444/93

\title{
The Electric Charge of Neutrinos and Plasmon Decay
}

\author{
T. Altherr \\ Theory Division, CERN, CH-1211 Geneva 23, Switzerland \\ and \\ P. Salati \\ ENSLAPP, BP110, F-74941 Annecy-le-Vieux Cedex, France \\ Institut universitaire de France
}

\begin{abstract}
By using both thermal field theory and a somewhat more intuitive method, we define the electric charge as well as the charge radius of neutrinos propagating inside a plasma. We show that electron neutrinos acquire a charge radius of order $\sim 6.5 \times$ $10^{-16} \mathrm{~cm}$, regardless of the properties of the medium. Then, we compute the rate of plasmon decay which such an electric charge or a charge radius implies. Taking into account the relativistic effects of the degenerate electron gas, we compare our results to various approximations as well as to recent calculations and determine the regimes where the electric charge or the charge radius does mediate the decay of plasmons. Finally, we discuss the stellar limits on any anomalous charge radius of neutrinos.
\end{abstract}

CERN-TH-7076/93

November 93

${ }^{*}$ On leave of absence from L.A.P.P., BP 110, F-74941, Annecy-le-Vieux Cedex, France 


\section{Introduction}

The plasmon decay process is an important cooling mechanism for stars whose core is degenerate. It is particularly relevant as regards the evolution of young white dwarves and red giant stars. In these two stellar objects, the core is composed of degenerate matter, with density $\rho \simeq 10^{6} \mathrm{~g} \mathrm{~cm}^{-3}$ and temperature in the range from $T=10^{7}$ to $10^{9}$ $\mathrm{K}$. As a consequence of this degeneracy, all electronic levels below the Fermi sphere are filled up. A typical value of the electron momentum is $p_{F} \sim 400 \mathrm{keV}$, so that electrons are neither non-relativistic, nor ultra-relativistic.

The plasmon decay process was first considered a long time ago, in the pioneering work by Adams, Ruderman and Woo [1], which led to a rich literature [2]. The subject has recently been revived when it was realized that the plasmon dispersion relations, which were used previously, did not incorporate the relativistic behaviour of the electron plasma [3]. The latest works that deal with these effects are due to Itoh et al. [1], Braaten and Segel [5] and Haft, Raffelt and Weiss [6].

In this paper, we complete the previous analysis and present the calculation of the plasmon decay rate in the framework of two different but complementary approaches. The first discussion is based on considerations of thermal field theory while the second analysis is a more intuitive, kinetic theory-like, method. We show that both reasonings lead to the same answer. We also stress the important distinction between the effective electric charge which neutrinos exhibit when they couple to a classical electromagnetic field and their charge radius which may be strictly defined in the soft regime where the energy and momentum of plasmons are small.

The content of this work is as follows. In section 2, we derive the electric charge which a neutrino acquires when it propagates inside matter and interacts with a classical electromagnetic field. In section 3, we discuss the charge radius associated to the coupling between neutrinos and low-energy plasmons. We comment on these two phenomenological approximations which lead, a priori, to fairly different descriptions of the electromagnetic properties of neutrinos. In section 4, we present the analytic expressions for the plasmon decay process. We discuss our numerical results for the case of red-giants and white dwarves in section 5 where various approximations to the energy loss rate are presented along with their respective domain of validity. We finally conclude that, for

most astrophysical purposes, the charge radius and the electric charge descriptions of the neutrino behaviour, give indeed the same answer. 


\section{The neutrino electric charge and the hard regime}

When neutrinos propagate inside matter, they scatter coherently on charged particles, such as the electrons and positrons of the thermal bath. This process is responsible for the "additional" inertia which neutrinos possess in a plasma [7]. Another possibility is that neutrinos diffuse coherently on electrons and positrons which, in turn, couple to the electromagnetic field $A^{\beta}[$ []. The Feynman graphs responsible for the latter reaction are presented in fig. 1 where both electrons (fig. 1a and 1b) and positrons (fig. 1c and 1d) have been taken into account. Note that the final electronic (or positronic) state is just the same as the incoming one, hence a modification of the electromagnetic coupling of neutrinos which appears directly at the level of the amplitude of this coherent diffusion. The effective coupling of neutrinos to electrons results from the low-energy limit of the Standard Glashow-Weinberg-Salam theory :

$$
\mathcal{L}=\frac{G_{F}}{\sqrt{2}}\left\{\bar{\Psi}_{\nu} \gamma_{\alpha}\left(1-\gamma_{5}\right) \Psi_{\nu}\right\} \quad\left\{\bar{\Psi}_{e} \gamma^{\alpha}\left(g_{V}-g_{A} \gamma_{5}\right) \Psi_{e}\right\} .
$$

Both $Z$ and $W$ exchanges are taken into account for electron neutrinos so that

$$
g_{V}=\frac{1}{2}+2 \sin ^{2} \theta_{W} \quad \text { and } \quad g_{A}=\frac{1}{2},
$$

whilst, for $\nu_{\mu}$ and $\nu_{\tau}$ where the $Z$ boson alone mediates the interaction, the coupling parameters are

$$
g_{V}=-\frac{1}{2}+2 \sin ^{2} \theta_{W} \quad \text { and } \quad g_{A}=-\frac{1}{2} .
$$

In order to compute the effective coupling of neutrinos to the electromagnetic field $A^{\beta}$ inside a plasma, the spectator electrons must be integrated out. Let us consider a volume $V$ of matter. The electronic state $\Psi_{e}$ describes the presence of a single electron of momentum $\vec{p}$ in the whole volume $V$, hence the normalisation

$$
\Psi_{e}(\vec{p}, s)=\sqrt{\frac{m}{V E}} u(\vec{p}, s),
$$

where $m$ and $E$ stand respectively for the mass and the energy of the electron. For positrons, $u(\vec{p}, s)$ is replaced by $v(\vec{p}, s)$. The electromagnetic coupling of neutrinos may therefore be expressed as a sum of the amplitude of the coherent diffusion pictured in fig. 1, over the electronic and positronic states of the thermal bath :

$$
\begin{aligned}
& \mathcal{L}_{\text {eff }}= \frac{G_{F}}{\sqrt{2}}\left\{\bar{\Psi}_{\nu} \gamma_{\alpha}\left(1-\gamma_{5}\right) \Psi_{\nu}\right\} A_{\beta} \int \frac{V d^{3} \vec{p}}{8 \pi^{3}} \sum_{s} \times \\
& \eta_{e}(\vec{p})\left\{\begin{array}{c}
{\left[\bar{\Psi}_{e}(\vec{p}, s) \gamma^{\alpha}\left(g_{V}-g_{A} \gamma_{5}\right) \frac{1}{(P+\not-m)} e \gamma^{\beta} \Psi_{e}(\vec{p}, s)\right]} \\
+\left[\bar{\Psi}_{e}(\vec{p}, s) e \gamma^{\beta} \frac{1}{(P-\varnothing-m)} \gamma^{\alpha}\left(g_{V}-g_{A} \gamma_{5}\right) \Psi_{e}(\vec{p}, s)\right]
\end{array}\right\}
\end{aligned}
$$




$$
-\eta_{\bar{e}}(\vec{p})\left\{\begin{array}{c}
{\left[\bar{\Psi}_{\bar{e}}(\vec{p}, s) e \gamma^{\beta} \frac{1}{(-P-Q-m)} \gamma^{\alpha}\left(g_{V}-g_{A} \gamma_{5}\right) \Psi_{\bar{e}}(\vec{p}, s)\right]} \\
+\left[\bar{\Psi}_{\bar{e}}(\vec{p}, s) \gamma^{\alpha}\left(g_{V}-g_{A} \gamma_{5}\right) \frac{1}{(-P+Q-m)} e \gamma^{\beta} \Psi_{\bar{e}}(\vec{p}, s)\right]
\end{array}\right\} .
$$

Here, $P^{\mu}=(E, \vec{p})$ denotes the electron (or positron) momentum whilst $Q^{\mu}=(\omega, \vec{q})$ refers to the plasmon. Since the initial and final states of the spectator electrons are the same, expression (5) may be written as a sum of traces. The statistical occupation numbers of electrons and positrons are respectively taken care of by the Fermi-Dirac functions $\eta_{e}(\vec{p})$ and $\eta_{\bar{e}}(\vec{p})$. The effective Lagrangian (5) becomes

$$
\begin{aligned}
\mathcal{L}_{\text {eff }} & =\frac{e G_{F}}{\sqrt{2}}\left\{\bar{\Psi}_{\nu} \gamma_{\alpha}\left(1-\gamma_{5}\right) \Psi_{\nu}\right\} A_{\beta} \int \tilde{d p} \times \\
\eta_{e}(\vec{p}) & \left\{\begin{array}{l}
\frac{\operatorname{Tr}\left[(P+m) \gamma^{\alpha}\left(g_{V}-g_{A} \gamma_{5}\right)(P+\not+m) \gamma^{\beta}\right]}{Q^{2}+2 P \cdot Q} \\
+ \\
-\eta_{\bar{e}}(\vec{p})\left\{\begin{array}{l}
\frac{\operatorname{Tr}\left[(P+m) \gamma^{\beta}(P-\not+m) \gamma^{\alpha}\left(g_{V}-g_{A} \gamma_{5}\right)\right]}{Q^{2}-2 P \cdot Q} \\
+
\end{array}\right. \\
\frac{\operatorname{Tr}\left[(P-m) \gamma^{\beta}(-P-\not+m) \gamma^{\alpha}\left(g_{V}-g_{A} \gamma_{5}\right)\right]}{Q^{2}+2 P \cdot Q}
\end{array}\right\},
\end{aligned}
$$

where $\tilde{d p}$ is the Lorentz-invariant differential element

$$
\tilde{d p}=\frac{1}{2 E} \frac{d^{3} \vec{p}}{8 \pi^{3}}
$$

After the reduction of the above-mentionned traces and some straightforward algebra, the effective coupling of neutrinos to the electromagnetic field may be expressed as

$$
\mathcal{L}_{e f f}=\frac{G_{F}}{\sqrt{2}}\left\{\bar{\Psi}_{\nu} \gamma_{\alpha}\left(1-\gamma_{5}\right) \Psi_{\nu}\right\} \Gamma^{\alpha \beta} A_{\beta}
$$

where the tensor $\Gamma^{\alpha \beta}$ may be separated into its symmetric and antisymmetric components :

$$
\begin{aligned}
\Gamma^{\alpha \beta} & =4 e g_{V} \int \tilde{d p}\left\{\eta_{e}(\vec{p})+\eta_{\bar{e}}(\vec{p})\right\}\left\{\frac{(P \cdot Q)\left(P^{\alpha} Q^{\beta}+P^{\beta} Q^{\alpha}\right)-(P \cdot Q)^{2} g^{\alpha \beta}-Q^{2} P^{\alpha} P^{\beta}}{(P \cdot Q)^{2}-\left(Q^{2} / 2\right)^{2}}\right\} \\
& -2 i e g_{A} \epsilon^{\alpha \beta \mu \nu} \int \tilde{d} p\left\{\eta_{e}(\vec{p})-\eta_{\bar{e}}(\vec{p})\right\}\left\{\frac{P_{\mu} Q_{\nu} Q^{2}}{(P \cdot Q)^{2}-\left(Q^{2} / 2\right)^{2}}\right\} .
\end{aligned}
$$

This effective coupling $\Gamma^{\alpha \beta}$ has been derived fairly intuitively. As pictured in fig. 1, the neutrino wave interacts coherently with the charged species of the plasma - for that matter 
mostly electrons and positrons since the contribution of nuclei is negligible. Expression (9) may be directly obtained from mere considerations of thermal field theory.

At the one-loop level, the Feynman rules for finite temperature field theory are quite simple [9]. The only ingredient is here the electron propagator, given by

$$
S_{F}(P)=(P+m)\left[\frac{i}{P^{2}-m^{2}+i \epsilon}-2 \pi \delta\left(P^{2}-m^{2}\right) N_{F}(P)\right]
$$

where the statistical factor $N_{F}(P)$ may be expressed as

$$
N_{F}(P)=\frac{\theta\left(P_{0}\right)}{e^{\left(P_{0}-\mu\right) / T}+1}+\frac{\theta\left(-P_{0}\right)}{e^{\left(-P_{0}+\mu\right) / T}+1} .
$$

Using the same conventions as before, the only non-vanishing contribution to the one-loop diagram of fig. 2 is

$$
\begin{aligned}
\Gamma^{\alpha \beta} & =e \int \frac{d^{4} P}{(2 \pi)^{3}} \operatorname{Tr}\left[\gamma^{\alpha}\left(g_{V}-g_{A} \gamma_{5}\right)(P+m) \gamma^{\beta}(P+\not+m)\right] \\
& \times\left\{\frac{\delta\left(P^{2}-m^{2}\right) N_{F}(P)}{(P+Q)^{2}-m^{2}}+\frac{\delta\left[(P+Q)^{2}-m^{2}\right] N_{F}(P+Q)}{P^{2}-m^{2}}\right\},
\end{aligned}
$$

which, after a change of variables, reduces to relation (9). The same result could also have been derived in the imaginary-time formalism.

The effective electric charge of the neutrinos which propagate inside a plasma may be defined as the limit of expression (9) where $A^{\beta}$ describes a classical electromagnetic field. The behaviour of finite temperature field theory and especially of its perturbative approach of interactions is quite rich. Inside matter, photons no longer propagate at the speed of light. On the contrary, they follow dispersion relations which imply the existence of longitudinal and transverse modes - see equations (29) and (32). The transverse photons are extremely similar to the ordinary quanta of the vacuum. They should be disentangled from the longitudinal modes which are mere collective excitations of the plasma. That is why the transverse branch alone exhibits an asymptote where the energy $\omega$ and the momentum $\vec{q}$ of plasmons reach up to infinity at constant $Q^{2}=\omega^{2}-q^{2}$. In this so-called hard limit [10], the transverse photons behave as if they propagated in the vacuum, with the non-vanishing mass $m_{T}$. Note that $Q^{2}$ is always of order $\omega_{0}^{2}$, where the plasma frequency $\omega_{0}$ is smaller than the electron mean energy $\left\langle E_{e}>\right.$ by a factor of $e=\sqrt{4 \pi \alpha}$, i.e., $\omega_{0} \sim e<E_{e}>\sim e T$ or $e p_{F}$. Therefore, in the hard regime, the components $\omega$ and $q$ may vary regardless of $Q^{2}$ which behaves as a small perturbation of order $\alpha$. In relation (9), expressions such as $(P \cdot Q)$ or $P^{\alpha} Q^{\beta}$ may be considered as leading contributions with respect to the correction $Q^{2} \sim O(\alpha)$. The tensor $\Gamma^{\alpha \beta}$ may therefore 
be expanded as a function of the small parameter $Q^{2}$, and the leading term turns out to be the symmetric expression

$$
\begin{aligned}
\Gamma^{\alpha \beta} & \simeq 4 e g_{V} \int \tilde{d p}\left\{\eta_{e}(\vec{p})+\eta_{\bar{e}}(\vec{p})\right\}\left\{\frac{P^{\alpha} Q^{\beta}+P^{\beta} Q^{\alpha}-(P \cdot Q) g^{\alpha \beta}}{P \cdot Q}\right\} \\
& + \text { terms of order } \alpha .
\end{aligned}
$$

As expression (13) is folded into the vertex (8), it further simplifies. In the Lorentz gauge where $\partial_{\beta} A^{\beta}=0$, the contribution $Q^{\beta} A_{\beta}$ vanishes. Note that the effective interaction (8) is gauge invariant since $\Gamma^{\alpha \beta} Q_{\alpha}=\Gamma^{\alpha \beta} Q_{\beta}=0$, as is obvious from expression (9). Therefore, restricting ourselves to the Lorentz gauge does not alter the general import of our reasoning. Finally, neutrinos may be considered as massless species, a fairly good approximation since plasmon decay merely involves the electron neutrino for which $g_{V} \simeq 1$ and whose mass is orders of magnitude smaller than the typical energies at stellar cores. Since expression $\bar{\Psi}_{\nu} Q\left(1-\gamma_{5}\right) \Psi_{\nu}$ vanishes in that limit, the neutrino coupling further simplifies into the effective Lagrangian :

$$
\mathcal{L}_{e f f}=-e Q_{\nu}\left\{\bar{\Psi}_{\nu} \gamma^{\beta} L \Psi_{\nu}\right\} A_{\beta}
$$

The left-helicity operator $L$ is defined as $L=\left(1-\gamma_{5}\right) / 2$. The neutrino behaves as if it had the electric charge

$$
Q_{\nu}=4 \sqrt{2} G_{F} g_{V} \mathcal{I}_{P}
$$

This charge depends on the properties of the plasma through the integral

$$
\mathcal{I}_{P}=\frac{1}{4 \pi^{2}} \int \frac{p^{2} d p}{E}\left\{\eta_{e}(p)+\eta_{\bar{e}}(p)\right\}
$$

In a degenerate gas of electrons, a typical situation inside red-giants or white dwarves, this plasma integral over the statistical distribution of electrons may be expressed as

$$
\mathcal{I}_{P}=\frac{p_{F} E_{F}}{8 \pi^{2}}\left\{1+\left(\frac{v_{F}^{2}-1}{2 v_{F}}\right) \ln \left(\frac{1+v_{F}}{1-v_{F}}\right)\right\},
$$

where $v_{F}=p_{F} / E_{F}$ denotes the velocity at the surface of the Fermi sea. At the helium core of red-giant stars, for instance, the density reaches up to $\sim 10^{6} \mathrm{~g} \mathrm{~cm}^{-3}$. The Fermi momentum is $p_{F} \sim 410 \mathrm{keV}$, hence an effective electric charge $Q_{\nu} \sim 6.4 \times 10^{-14}$ which, in spite of being some 13 orders of magnitude smaller than the charge of the electron, induces a significant energy loss, so large that it dominates the evolution of stars during their ascension of the red-giant branch. 


\section{The neutrino charge radius and the soft limit}

In the low energy part of the transverse branch as well as all along the longitudinal branch, the energy $\omega$ and the momentum $q$ of plasmons are much smaller than the average energy of the charged species of the thermal bath. In this so-called soft regime [10] where $q$ is small, the components $Q^{\mu}$ of the plasmon four-momentum may be considered of order $e=\sqrt{4 \pi \alpha}$ with respect to the typical electron momentum, i.e.,

$$
\frac{\left|Q^{\mu}\right|}{\left|P^{\nu}\right|} \sim \mathcal{O}(e) \sim \mathcal{O}(\sqrt{4 \pi \alpha}) \ll 1 .
$$

Notice that in the hard regime, $Q^{2}$ is of order $\mathcal{O}(\alpha)$ whereas both components $Q^{\mu}$ and $P^{\nu}$ are leading order terms. On the contrary, in the soft regime which we discuss now, expressions such as $\omega, q$ or $\sqrt{\omega^{2}-q^{2}}$ are of order $\mathcal{O}(e)$, so that the perturbative expansion of expression (9) is now quite different than in the hard-loop approximation. In particular, the axial part of the tensor $\Gamma^{\alpha \beta}$ is of order $\mathcal{O}(e)$. However, it does not interfere with the vector component of $\Gamma^{\alpha \beta}$ as regards plasmon decay, and therefore contributes a term only of order $\mathcal{O}(\alpha)$ which we will not consider in what follows. The leading contribution to $\Gamma^{\alpha \beta}$ is the vector component :

$$
\begin{aligned}
\Gamma^{\alpha \beta} & =4 e g_{V} \int \tilde{d p}\left\{\eta_{e}(\vec{p})+\eta_{\bar{e}}(\vec{p})\right\}\left\{\frac{(P \cdot Q)\left(P^{\alpha} Q^{\beta}+P^{\beta} Q^{\alpha}\right)-(P \cdot Q)^{2} g^{\alpha \beta}-Q^{2} P^{\alpha} P^{\beta}}{(P \cdot Q)^{2}}\right\} \\
& + \text { terms of order } e .
\end{aligned}
$$

As noticed already some thirty years ago [1], the previous expression is merely the polar-

ization tensor $\Pi^{\alpha \beta}$ associated to the propagation of electromagnetic waves inside a plasma, i.e.,

$$
\Gamma^{\alpha \beta}=\frac{g_{V}}{e} \Pi^{\alpha \beta}
$$

hence the effective Lagrangian

$$
\mathcal{L}_{e f f}=\frac{\sqrt{2} G_{F} g_{V}}{e}\left\{\bar{\Psi}_{\nu} \gamma_{\alpha} L \Psi_{\nu}\right\} \Pi^{\alpha \beta} A_{\beta}
$$

where the electromagnetic field couples to the neutrino current through the polarization tensor. Note that $\Pi^{\alpha \beta}$ may be broken up into the operators $P_{T}^{\alpha \beta}$ and $P_{L}^{\alpha \beta}$ which respectively project the polarization $A^{\beta}$ on the transverse and longitudinal modes [11 :

$$
\Pi^{\alpha \beta}=\Pi_{T}(\omega, \vec{q}) P_{T}^{\alpha \beta}+\Pi_{L}(\omega, \vec{q}) P_{L}^{\alpha \beta}
$$

Since the plasmon four-momentum follows the dispersion relation

$$
Q^{2}+\Pi_{T, L}(\omega, \vec{q})=0
$$


expression (21) simplifies further into :

$$
\mathcal{L}_{e f f}=-\frac{\sqrt{2} G_{F} g_{V}}{e} Q^{2}\left\{\bar{\Psi}_{\nu} \gamma^{\beta} L \Psi_{\nu}\right\} A_{\beta}
$$

Therefore, in the soft regime, neutrinos interact with the electromagnetic field as if they had a non-vanishing charge radius :

$$
\mathcal{L}_{\text {eff }}=-e \frac{<r^{2}>}{6} Q^{2}\left\{\bar{\Psi}_{\nu} \gamma^{\beta} L \Psi_{\nu}\right\} A_{\beta}
$$

with the plasmon four-momentum $Q^{2}$ factored out in the coupling, as is typical. This thermal charge radius, which neutrinos built from their coherent diffusion on the spectator electrons of the plasma, may be expressed as

$$
<r^{2}>=\frac{3 G_{F}}{\sqrt{2} \pi \alpha} g_{V}
$$

and may be understood as the superimposition of two distributions of opposite electric charge, with same centers, but with different spatial extensions. By surfing on the electrons of the plasma, the neutrino behaves as if it acquired an inner structure whose typical size is surprisingly independent of the properties of the thermal bath :

$$
<r^{2}>\simeq\left(6.5 \times 10^{-16} \mathrm{~cm}\right)^{2} g_{V}
$$

We therefore conclude that, in the soft limit, neutrinos interact with the electromagnetic field through a charge radius coupling whereas, in the hard regime, they possess an effective electric charge.

In a degenerate medium, the plasma frequency $\omega_{0}$ is related to the Fermi momentum $p_{F}$

$$
\omega_{0}^{2}=\frac{4 \alpha}{3 \pi} \frac{p_{F}^{3}}{E_{F}}
$$

A fully relativistic treatment of the electron bath leads to the dispersion relation for the longitudinal mode [5, 12] :

$$
\omega_{L}^{2}=\omega_{0}^{2}\left(\frac{3 \omega_{L}^{2}}{v_{F}^{2} q^{2}}\right)\left\{\left(\frac{\omega_{L}}{2 v_{F} q}\right) \ln \left(\frac{\omega_{L}+v_{F} q}{\omega_{L}-v_{F} q}\right)-1\right\},
$$

where $v_{F}=p_{F} / E_{F}$ stands for the velocity at the surface of the Fermi sea. The energy and the momentum of plasmons are respectively denoted by $\omega_{L}$ and $q$. The longitudinal branch extends up to the point where it crosses the light cone, for

$$
q_{\max }=\left\{\frac{3}{v_{F}^{2}}\left(\frac{1}{2 v_{F}} \ln \left[\frac{1+v_{F}}{1-v_{F}}\right]-1\right)\right\}^{1 / 2} \omega_{0} .
$$


As $q$ increases from 0 to $q_{\text {max }}$, the four-momentum $Q^{2}$ steadily decreases from the plasma frequency $\omega_{0}^{2}$ down to 0 . Note that $\omega_{L}$ and $q$ cannot reach up to infinity, except in the ultra-relativistic case where, nevertheless, they cannot vary regardless of the value of $Q^{2}$. The longitudinal branch corresponds therefore to the soft regime. In particular, when electrons are non-relativistic, i.e., for vanishing $v_{F}$, relation (29) simplifies into

$$
\omega_{L}=\omega_{0}
$$

and the longitudinal branch extends up to $q_{\max }=\omega_{0}$. The non-relativistic regime is a good example of soft behaviour in so far as $\omega_{L}$ and $q$, which cannot exceed $\omega_{0}$, are obviously of order $\mathcal{O}(e)$ with respect to the Fermi momentum $p_{F}$. As regards the transverse mode, the dispersion relation inside degenerate matter may be expressed directly as a function of the effective plasmon mass :

$$
\omega_{T}^{2}-q^{2}=\omega_{0}^{2}\left(\frac{3 \omega_{T}^{2}}{2 v_{F}^{2} q^{2}}\right)\left\{1+\left(\frac{v_{F}^{2} q^{2}}{\omega_{T}^{2}}-1\right)\left(\frac{\omega_{T}}{2 v_{F} q}\right) \ln \left(\frac{\omega_{T}+v_{F} q}{\omega_{T}-v_{F} q}\right)\right\} .
$$

The low-energy part of the transverse branch corresponds to the soft regime, whereas the hard limit is recovered for $\omega_{T} \simeq q \rightarrow+\infty$. When the hard regime obtains, transverse plasmons behave as if they propagated with the effective mass

$$
m_{T}^{2}=\left(\frac{3 \omega_{0}^{2}}{2 v_{F}^{2}}\right)\left\{1+\left(\frac{v_{F}^{2}-1}{2 v_{F}}\right) \ln \left(\frac{1+v_{F}}{1-v_{F}}\right)\right\} .
$$

As $q$ increases along the transverse branch, note that the four-momentum $Q^{2}$ raises from $\omega_{0}^{2}$ to $m_{T}^{2}$. As is clear from relations (17), (28) and (33), this asymptotic mass may be readily expressed as a function of the integral $\mathcal{I}_{P}$ which dominates the behaviour of the neutrino electric charge $Q_{\nu}$ :

$$
m_{T}^{2}=4 e^{2} \mathcal{I}_{P}
$$

When the stellar plasma is ultra-relativistic, the transverse mass of high-energy (i.e., hard) plasmons is $m_{T}=\sqrt{3 / 2} \omega_{0}$. On the contrary, for non-relativistic electrons, relation (32) becomes

$$
\omega_{T}^{2}=\omega_{0}^{2}+q^{2}
$$

and plasmons propagate with the mass $\omega_{0}$ all along the transverse branch, even in its soft region.

Both longitudinal and transverse dispersion relations were first derived by Jancovici 113, but had a much more complicated form. It was realized later that the simplified expressions (29) and (32) could be used in a much wider regime [5, 12].

A thorough inspection of relation (9) shows that equation (13) behaves as the mere limit of equation (19) for vanishing $Q^{2}$, at fixed plasmon momentum. The transition 
between the soft and hard behaviours is therefore smooth. The expressions derived in this section are surprisingly more general than what could be naively guessed. Not only are they valid in the soft limit, but they cover the entire plasmon spectrum and, as a consequence, the hard regime as well. The effective charge radius is a good description of the electromagnetic properties of neutrinos. To illustrate this point, let us consider the effective interaction (24) which has been strictly derived in the soft regime. For high energy plasmons of the transverse mode, $Q^{2}$ may be replaced by $4 e^{2} \mathcal{I}_{P}$ so that the charge radius coupling $\left(<r^{2}>Q^{2} / 6\right)$ translates into the electric charge $Q_{\nu}$ of relation (15). The charge radius, which has been defined in the soft regime, pleasantly behaves as an effective electric charge when the hard limit is taken. More generally, it translates, along the asymptote of the transverse branch, into the electric charge

$$
Q_{\nu}=\frac{G_{F} g_{V}}{2 \sqrt{2} \pi \alpha} m_{T}^{2}
$$

The Lagrangian (24) provides therefore a comprehensive description of the electromagnetic properties of neutrinos propagating inside a plasma, valid for the entire spectrum of both longitudinal and transverse modes. It reduces to an electric charge when the hard regime applies, i.e., in the asymptotic limit of the transverse branch where plasmons behave as a classical field.

\section{The plasmon decay rate}

Inside stars, plasma waves may decay into neutrino pairs as a result of the effective coupling (24). At the helium cores of red-giants where the stellar density is fairly large, the plasma frequency reaches up to $\omega_{0} \sim 20 \mathrm{keV}$. Once produced, the neutrinos do not interact with stellar interiors. Most of the stars are indeed completely transparent to these species which, therefore, escape freely from their cores. The corresponding cooling rate depends on the distribution of plasma waves as well as on the efficiency with which a plasmon is transmuted into a neutrino pair.

The decay rate of plasmons obtains from the phase space integral

$$
\Gamma_{D}=\frac{1}{2 \omega} \int \tilde{d k_{\nu}} \tilde{d k}_{\bar{\nu}}(2 \pi)^{4} \delta^{4}\left(Q-k_{\nu}-k_{\bar{\nu}}\right) \sum_{\text {spins }}\left|\mathcal{M}\left(\gamma_{Q} \rightarrow \nu \bar{\nu}\right)\right|^{2}
$$

where the relevant matrix element is

$$
\mathcal{M}\left(\gamma_{Q} \rightarrow \nu \bar{\nu}\right)=\frac{\sqrt{2} G_{F} g_{V}}{e} Q^{2}\left\{\bar{U}\left(k_{\nu}\right) \notin L V\left(k_{\bar{\nu}}\right)\right\} .
$$

In the Lorentz gauge, the polarization of transverse plasmons are the pure vectors $\overrightarrow{\epsilon_{T}}$, transverse to the direction $\vec{q}$ of motion, whilst the longitudinal polarization is taken care 
of by the four-vector

$$
\epsilon_{L}^{\mu}=\left(q, \frac{\vec{q}}{q} \omega_{L}\right) / \sqrt{\omega_{L}^{2}-q^{2}} .
$$

A straightforward calculation leads to the decay rate

$$
\Gamma_{D}=\frac{1}{48 \pi^{2}} \frac{G_{F}^{2} g_{V}^{2}}{\alpha} \frac{1}{\omega}\left(Q^{2}\right)^{3} .
$$

In the hard regime, where the electromagnetic properties of neutrinos are well described by the electric charge $e Q_{\nu}$ - see relations (14) and (15) - the decay rate becomes

$$
\Gamma_{D}=\frac{\alpha}{6} Q_{\nu}^{2} \frac{Q^{2}}{\omega} .
$$

The neutrino energy loss may be derived from the sum over the Bose-Einstein distribution $\eta_{B}(\omega)$ of the individual decays

$$
\epsilon_{\text {Loss }}=\int \frac{d^{3} \vec{q}}{8 \pi^{3}} g_{h} \eta_{B}(\omega) \omega Z \Gamma_{D} .
$$

Each neutrino pair carries outside the star the energy $\omega$ of its progenitor. The helicity factor $g_{h}$ respectively takes the values 1 and 2 for the longitudinal and transverse modes. The additional factor $Z$ corrects for the term $1 / 2 \omega$ in relation (37). For ordinary particles with mass $m$, the relation between the energy $E$ and the momentum $\vec{p}$ is merely

$$
E^{2}-\vec{p}^{2}=P^{2}=m^{2},
$$

so that, when the Lorentz invariant expression $d^{4} P \delta\left(P^{2}-m^{2}\right)$ is integrated over the positive values of $P^{0}$, it translates into the conventional phase space element $d^{3} \vec{p} / 2 E$. For plasmons, expression (43) is replaced by the more intricate dispersion relations (23) which, once integrated over the positive values of $Q^{0}$, become

$$
\int d^{4} Q \theta\left(Q^{0}\right) \delta\left\{Q^{2}+\Pi\left(Q^{0}, \vec{q}\right)\right\}=Z \frac{d^{3} \vec{q}}{2 \omega},
$$

with

$$
Z^{-1}=\left|1+\frac{\partial \Pi}{\partial \omega^{2}}\right|
$$

For the transverse mode of a degenerate plasma, this normalisation factor may be expressed as

$$
Z_{T}=\frac{2 \omega_{T}^{2}\left(\omega_{T}^{2}-v_{F}^{2} q^{2}\right)}{3 \omega_{0}^{2} \omega_{T}^{2}+\left(\omega_{T}^{2}+q^{2}\right)\left(\omega_{T}^{2}-v_{F}^{2} q^{2}\right)-2 \omega_{T}^{2}\left(\omega_{T}^{2}-q^{2}\right)},
$$

whereas, for the longitudinal branch, it becomes

$$
Z_{L}=\frac{2 \omega_{L}^{2}}{Q^{2}}\left\{\frac{\left(\omega_{L}^{2}-v_{F}^{2} q^{2}\right)}{3 \omega_{0}^{2}-\left(\omega_{L}^{2}-v_{F}^{2} q^{2}\right)}\right\} .
$$


In the non-relativistic regime for which the velocity $v_{F} \rightarrow 0, Z_{T}$ merely reduces to unity whereas $Z_{L}$ tends towards $\omega_{0}^{2} / Q^{2}$.

Once again, similar expressions for the energy loss may be obtained in the thermal field theory approach. The neutrino (antineutrino) production rate, due for instance to transverse photon decay, is easily derived by using the cutting rules of Kobes and Semenoff [14, given by

$$
\begin{aligned}
R_{T}= & \frac{d N_{\nu}}{d^{4} x} \\
= & \frac{2 G_{F}^{2} g_{V}^{2}}{e^{2}} \int \frac{d^{4} Q}{(2 \pi)^{4}} \int \frac{d^{4} K}{(2 \pi)^{4}} 2 \pi \delta\left(K^{2}\right) 2 \pi \delta\left[(Q-K)^{2}-m^{2}\right]\left\{\eta_{B}(\omega)+\theta\left(-Q^{0}\right)\right\} \\
& \times 2 \pi \delta\left[Q^{2}-\operatorname{Re} \Pi_{T}(Q)\right] \operatorname{Tr}\left[K \gamma^{\mu}(K+Q) \gamma^{\nu}\right] \mathcal{P}_{T}^{\mu \nu}\left(Q^{2}\right)^{2}
\end{aligned}
$$

Here $K$ and $Q$ are respectively the neutrino and photon four-momenta. The transverse photon projection operator is

$$
\mathcal{P}_{T}^{i j}=-\delta^{i j}+q^{i} q^{j} / q^{2}
$$

with all its other components set equal to zero [11]. After performing the integrations, the result takes the final form

$$
R_{T}=\frac{G_{F}^{2} g_{V}^{2}}{48 \pi^{4} \alpha} \int_{0}^{\infty} \frac{q^{2} d q}{\omega} Z_{T} \eta_{B}(\omega)\left(Q^{2}\right)^{3}
$$

The energy loss rate may be inferred directly from the previous integral :

$$
\epsilon_{T}=\frac{G_{F}^{2} F_{\nu}}{48 \pi^{4} \alpha} \int_{0}^{\infty} q^{2} d q Z_{T} \eta_{B}(\omega)\left(Q^{2}\right)^{3}
$$

where the factor $F_{\nu}$ takes into account the three neutrino species produced in plasmon decay

$$
F_{\nu}=\sum_{\nu} g_{V}^{2}(\nu)=\frac{3}{4}-2 \sin ^{2} \theta_{W}+12 \sin ^{4} \theta_{W}
$$

As regards the longitudinal branch, a similar expression obtains where the plasmon momentum is integrated up to $q_{\max }$

$$
\epsilon_{L}=\frac{G_{F}^{2} F_{\nu}}{96 \pi^{4} \alpha} \int_{0}^{q_{\max }} q^{2} d q Z_{L} \eta_{B}(\omega)\left(Q^{2}\right)^{3}
$$

In the next section, the behaviour of the exact cooling rate $\epsilon=\epsilon_{T}+\epsilon_{L}$ will be discussed in various stellar environments. Two approximations of this exact energy loss may be defined :

(i) The estimate $\epsilon^{Q}$ obtains when the charge radius (25) is replaced by the electric charge (14), so that the transverse cooling is now

$$
\epsilon_{T}^{Q}=\frac{16 \alpha G_{F}^{2} F_{\nu}}{3 \pi^{2}} \mathcal{I}_{P}^{2} \int_{0}^{\infty} q^{2} d q Z_{T} \eta_{B}(\omega) Q^{2}
$$


whereas the longitudinal energy loss is

$$
\epsilon_{L}^{Q}=\frac{8 \alpha G_{F}^{2} F_{\nu}}{3 \pi^{2}} \mathcal{I}_{P}^{2} \int_{0}^{q_{\max }} q^{2} d q Z_{L} \eta_{B}(\omega) Q^{2}
$$

(ii) The non-relativistic approximation corresponds to the limit where $v_{F}$ vanishes. The plasma frequency is therefore defined as the ratio

$$
\omega_{0}^{2}=\frac{4 \alpha}{3 \pi} \frac{p_{F}^{3}}{m}
$$

As the dispersion relations (31) and (35) now apply, the expressions for the energy loss rate $\epsilon^{N R}$ considerably simplify. We simply recover the results of Adams, Ruderman and Woo [1], later on corrected by Zaidi [2]. Neutrino emission by transverse photons occurs at a pace :

$$
\epsilon_{T}^{N R}=\frac{G_{F}^{2} F_{\nu}}{48 \pi^{2} \alpha} \omega_{0}^{6} n_{\gamma}
$$

where the photon density $n_{\gamma}$ is defined by

$$
n_{\gamma}=\frac{1}{\pi^{2}} \int_{0}^{+\infty} q^{2} d q \eta_{B}\left(\omega_{T}\right)
$$

As regards the energy dragged away from the longitudinal mode, the corresponding loss rate may be expressed exactly

$$
\epsilon_{L}^{N R}=\frac{G_{F}^{2} F_{\nu}}{1260 \pi^{4} \alpha} \omega_{0}^{9} \eta_{B}\left(\omega_{0}\right)
$$

\section{Discussion}

Neutrino cooling dominates the slow contraction of red-giant stars [15], whose core temperature steadily increases in time, up to the point where, at the tip of the red-giant branch of the Hertzsprung-Russell diagram, the triple-alpha reaction sets on. For low-mass stars, typical conditions at the center are $\rho \sim 10^{6} \mathrm{~g} \mathrm{~cm}^{-3}$ with $T \simeq 10^{8} \mathrm{~K}$. During this stage of their evolution, stars have a burning shell of hydrogen surrounding a degenerate helium core whose mass increases at a pace dominated by the neutrino losses. Thus, the mass of helium processed on the red-giant branch may be related to plasmon decay and its asso-

ciated cooling [16]. As a matter of fact, observations of the color-magnitude diagrams of globular clusters offer an interesting handle on the cooling of red-giant cores by neutrino emission. The latter mechanism also dominates the early evolution of white-dwarves, slightly after their formation, when they are still relatively hot, with central temperature $T \sim 10^{7}$ to $10^{9} \mathrm{~K}$. The cooling of white-dwarves is expected to be measured in the near 
future, using asteroseismology techniques. The slow variation of the period of pulsating white-dwarves may be directly related to the decrease of their central temperature [17].

The case of red-giant stars is embraced in fig. 3a where the exact energy loss rate $\epsilon$ varies with the density $\rho$, at fixed temperature $T=10^{8} \mathrm{~K}$ and fixed electron fraction $Y_{e}=0.5$ (pure helium). For small values of the density $\rho$, the Fermi momentum

$$
p_{F}=\left(3 \pi^{2} Y_{e} \rho / m_{u}\right)^{1 / 3}
$$

is smaller than the electron mass $m$, and the non-relativistic regime applies. The point $\rho=10^{5} \mathrm{~g} \mathrm{~cm}^{-3}$, for instance, corresponds to $p_{F}=190 \mathrm{keV}$, so that the plasma frequency is $\omega_{0} \simeq 6 \mathrm{keV}$. Even at such a low density, electrons are degenerate because the temperature $T \simeq 9 \mathrm{keV}$ is negligible with respect to the Fermi momentum $p_{F}$. Since $T$ exceeds the plasma frequency $\omega_{0}$ in the left-hand side region of fig. 3a, relations (57) and (59) simplify respectively into

$$
\epsilon_{T}^{N R}\left(T>\omega_{0}\right)=\frac{\zeta(3)}{24 \pi^{4} \alpha} G_{F}^{2} F_{\nu} \omega_{0}^{6} T^{3}
$$

and

$$
\epsilon_{L}^{N R}\left(T>\omega_{0}\right)=\frac{G_{F}^{2} F_{\nu}}{1260 \pi^{4} \alpha} \omega_{0}^{8} T
$$

The transverse rate overshadows completely the longitudinal emission. Note that at low density, the energy loss $\epsilon \simeq \epsilon_{T}^{N R}+\epsilon_{L}^{N R}$ increases with $\rho$. On the contrary, in the high density regime (right-hand side domain of fig. 3a), electrons are ultra-relativistic and the temperature is now much smaller than the plasma frequency, hence a decrease of $\epsilon$ with $\rho$. The Boltzmann factor $\exp \left(-\omega_{0} / T\right)$ drops as there are fewer and fewer plasmons. The maximum which the curve exhibits corresponds to the transition between these two regimes.

In fig. 4a, devoted to white-dwarf cooling, the density has been set equal to the typical value of 1.8 tons $\mathrm{cm}^{-3}$, a pure carbon composition has been assumed $\left(Y_{e}=0.5\right)$, and the temperature is varied from $10^{6}$ up to $10^{9} \mathrm{~K}$. The Fermi momentum $p_{F} \sim 500 \mathrm{keV}$ corresponds to the relativistic velocity $v_{F}=0.7$ and to the plasma frequency $\omega_{0}=23.2$ $\mathrm{keV}$. Note also that the transverse mass of high-energy photons is $m_{T}=24.7 \mathrm{keV}$. Since the maximal temperature considered here, $T=86 \mathrm{keV}$, is much smaller than the Fermi momentum, the gas is still degenerate. The increase of the energy loss rate $\epsilon$ with $T$ is impressive.

The conditions of density and temperature in the plots $3 \mathrm{~b}$ and $4 \mathrm{~b}$ are respectively the same as in fig. 3a and 4a. The solid line stands for the ratio $\epsilon^{Q} / \epsilon$ and corresponds to the electric charge approximation. The short dash curve refers to the non-relativistic estimate $\epsilon^{N R} / \epsilon$. The numerical fit published by Itoh et al. [4] is extensively used for astrophysical 
purposes. Its ratio to the exact cooling rate is represented here by the dotted curve while the short dash-dotted line stands for the recent numerical estimate by Haft et al. [6]. The energy loss rate $\epsilon$ is a function of both the density $\rho$ and the temperature $T$. In a degenerate medium, which is the situation under scrutiny here, the density alone determines the Fermi momentum, the plasma frequency as well as the dispersion relations of the plasmon propagation. Therefore, the structure of both transverse and longitudinal modes depends only on the parameter $\rho$. As for the temperature, it conditions the filling of the plasma states along the various branches and governs, for instance, the abovementionned density $n_{\gamma}$. Some remarks are in order.

(A) In the regime where the temperature exceeds the plasma frequency, the highenergy tail of both transverse and longitudinal branches are filled up. Note that $\omega_{L}{ }^{2}-q^{2}$ vanishes near the tip of the longitudinal branch. Therefore, the decays of longitudinal plasmons are suppressed and neutrino cooling is dominated by the behaviour of the highenergy, i.e., hard, transverse photons. The loss rate $\epsilon^{Q}$ is a good estimate of $\epsilon$ in the high temperature regime. That is why the ratio $\epsilon^{Q} / \epsilon$ (solid curve) becomes unity in the left-hand side of fig. $3 \mathrm{~b}$ and in the right-hand portion of fig. $4 \mathrm{~b}$.

(B) At low temperature, i.e., in the limit where $\omega_{0}$ exceeds $T$, the low-energy plasma states only are thermally excited, and the average momentum $q$ is, a priori, much smaller than the plasma frequency. Plasmons may be pictured as if they were at rest, with energy $\omega_{0}$, vanishing momentum $q$ and therefore an effective mass of order the plasma frequency $\omega_{0}$. The exact decay rate (40) varies as $\left(Q^{2}\right)^{3}$ whereas the approximate relation (41) is proportional to $m_{T}^{4} Q^{2}$. Therefore, when $T$ is small with respect to $\omega_{0}$, the ratio $\epsilon^{Q} / \epsilon$ behaves roughly as $\left(m_{T} / \omega_{0}\right)^{4}$. In fig. $3 \mathrm{~b}$ for instance, the solid curve tends asymptotically towards the ultra-relativistic limit of $(3 / 2)^{2}=2.25$ when $\rho$ is large. In the low temperature part of fig. $4 \mathrm{~b}$, it converges towards the value $(24.7 / 23.2)^{4} \simeq 1.3$ (see above). There is nevertheless an exception to this behaviour. When the plasma is non-relativistic, the longitudinal branch is noticeably flat and, unlike for the other mode, all its propagation states are equally filled up, even at extremely low temperature. The momentum $q$ is not forced to vanish because the Bose-Einstein factor $\eta_{B}\left(\omega_{0}\right)$ is constant throughout the longitudinal branch. In this regime, the energy loss

$$
\epsilon_{L}^{N R}\left(T<\omega_{0}\right)=\frac{G_{F}^{2} F_{\nu}}{1260 \pi^{4} \alpha} \omega_{0}^{9} e^{-\omega_{0} / T}
$$

eclipses completely the transverse production of neutrinos

$$
\epsilon_{T}^{N R}\left(T<\omega_{0}\right)=\frac{G_{F}^{2} F_{\nu}}{48 \sqrt{2} \pi^{7 / 2} \alpha} \omega_{0}^{15 / 2} T^{3 / 2} e^{-\omega_{0} / T} .
$$

Therefore, at low temperature and for a non-relativistic degenerate electron gas, the electric charge approximation $\epsilon^{Q}$ overestimates the exact rate by a factor of $35 / 8 \simeq 4.4$. 
(C) At low density, the non-relativistic regime obtains and $\epsilon^{N R}$ is a good estimate of the exact loss rate. Consequently, in the left-hand side of fig. $3 \mathrm{~b}$, the short dash curve approaches unity. On the contrary, for large values of the density, the non-relativistic expression (56) overestimates slightly the plasma frequency $\omega_{0}$. In this regime, the loss rate $\epsilon$ behaves as $\exp \left(-\omega_{0} / T\right)$, hence the exponential drop which the ratio $\epsilon^{N R} / \epsilon$ exhibits as the density increases (right-hand side of fig. $3 \mathrm{~b}$ ) or as the temperature diminishes (left-hand side of fig. 4b).

(D) For red-giant stars, the fit which Haft et al. propose is impressively good. Notice how the short dash-dotted curve remarkably sticks to unity throughout fig. $3 \mathrm{~b}$. The Japanese approximation slightly underestimates the exact rate below $\rho=10^{6} \mathrm{~g} \mathrm{~cm}^{-3}$. As regards white-dwarf cooling, Haft's fit is still fairly good, especially between $10^{7}$ and $10^{9} \mathrm{~K}$. On the contrary, the result by Itoh et al. exhibits wiggles at the transition temperatures of the numerical estimate whose quality is not completely satisfactory. Furthermore, below $T=10^{7} \mathrm{~K}$, the corresponding dotted curve diverges whereas Haft's fit still gives an accurate prediction.

The coherent diffusion of neutrinos on the electrons of a plasma induces an effective interaction with the electromagnetic field. An intuitive approach as well as a more formal discussion performed in the framework of thermal field theory have been presented here. Both methods lead to the same conclusions. Neutrinos which propagate inside matter acquire an effective charge radius of order $6.5 \times 10^{-16} \mathrm{~cm} g_{V}^{1 / 2}$, regardless of the specific properties of the plasma. The description of the electromagnetic interactions of neutrinos by a charge radius, strictly valid in the limit of the soft-loop regime, turns out to be more general and may apply to the entire spectrum of plasmons. In particular, in the hard limit of high-energy transverse quanta, this neutrino charge radius translates into a mere electric charge. Since plasmon decay is an efficient mechanism of stellar energy loss when the cores of stars are hot, i.e., in a regime where the estimate $\epsilon^{Q}$ is accurate, the thermal electric charge $Q_{\nu}$ of neutrinos is therefore relevant to astrophysics. However minute as it may look, it is nevertheless responsible for the evolution of red-giant stars as well as for the early cooling of nascent white-dwarves.

Suppose finally that the tau neutrino has a large charge radius of order $6.5 \times 10^{-16}$ $\mathrm{cm}$. Plasmon decay into $\nu_{\tau}$ pairs would induce an extra neutrino emission comparable to the standard production. Observations of the magnitude shift between the tip of the red-giant branch and the horizontal sequence of the HR diagram preclude the existence of any additional neutrino cooling with a magnitude larger than the standard energy loss. We readily infer a limit of order $6-7 \times 10^{-16} \mathrm{~cm}$ on any anomalous charge radius. For electron and muon neutrinos, the laboratory constraints, of order $1-3 \times 10^{-16} \mathrm{~cm}$, are 
already more stringent [18]. However, for tau neutrinos, the stellar limit compares fairly well to the upper bound of $8-9 \times 10^{-16} \mathrm{~cm}$ derived from the ASP and CELLO experiments [19. 


\section{References}

[1] J. B. Adams, M. A. Ruderman and C. H. Woo, Phys. Rev. 129 (1963) 1383.

[2] M. H. Zaidi, Nuovo Cim. 40A (1965) 502; G. Beaudet, V. Petrosian and E. E. Salpeter, Ap. J. 150 (1967) 979; D. A. Dicus, Phys. Rev. D6 (1972) 941.

[3] E. Braaten, Phys. Rev. Lett. 66 (1991) 1655.

[4] N. Itoh, H. Mutoh, A. Hikita and Y. Kohyama, Ap. J. 395 (1992) 622. See also H. Munakata, Y. Kohyama and N. Itoh, Ap. J. 296 (1985) 197.

[5] E. Braaten and D. Segel, Phys. Rev. D47 (1993) 1478.

[6] M. Haft, G. Raffelt and A. Weiss, to appear in Ap. J.

[7] D. Notzold and G. Raffelt, Nucl. Phys. B307 (1988) 924; P. B. Pal and T. N. Pham, Phys. Rev. D40 (1989) 259.

[8] A. Grau and J. A. Grifols, Phys. Lett. B166 (1986) 233; V. N. Oraevskii and V. B. Semikoz, Physica 142A (1987) 135; V. N. Oraevskii and V. N. Ursov, Phys. Lett. B209 (1988) 83; J. C. D’Ollivo, J. F. Nieves and P. B. Pal, Phys. Rev. D40 (1989) 3679; T. Altherr and K. Kainulainen, Phys. Lett. B262 (1991) 79; J. F. Nieves and P. B. Pal, Univ. of Texas preprint, CPP-93-12.

[9] N. P. Landsman and Ch. G. van Weert, Phys. Rep. 145 (1987) 141.

[10] E. Braaten and R. Pisarski, Nucl. Phys. B337 (1990) 569; Nucl. Phys. B339 (1990) 310.

[11] H. A. Weldon, Phys. Rev. D26 (1982) 1394.

[12] T. Altherr, E. Petitgirard and T. del Rio Gaztelurrutia, Astropart. Phys. 1 (1993) 289.

[13] B. Jancovici, Nuovo Cim. 25 (1962) 428.

[14] H. A. Weldon, Phys. Rev. D28 (1983) 2007; R. L. Kobes and G. W. Semenoff, Nucl. Phys. B260 (1985) 714 and B272 (1986) 329; N. Ashida, H. Nakkagawa, A. Niégawa and H. Yokota, Ann. Phys. (NY) 215 (1992) 315.

[15] G. Raffelt, Ap. J. 365 (1990) 559.

[16] A. V. Sweigart and P. G. Gross, Astrophys. J. Suppl. 36 (1978) 405. 
[17] S. O. Kepler et al., Ap. J. 378 (1991) L45. See also the analysis by J. Isern, M. Hernanz and E. Garcia-Berro, Ap. J. 392 (1992) L23.

[18] K. Abe et al., Phys. Rev. Lett. 58 (1987) 636; J. Dorenbosch et al., Zeit. fur Phys. C41 (1989) 567; L. A. Ahrens et al., Phys. Rev. D41 (1990) 3297; I. I. Gurevich et al., Kurchatov Institute of Atomic Energy preprint (1993).

[19] CELLO Collaboration, H. J. Behrend et al., Phys. Lett. B215 (1988) 186; C. Hearty et al., Phys. Rev. D39 (1989) 3207. 


\section{Figure Caption}

Fig. 1 The neutrino current diffuses coherently on the electrons (1a and 1b), and on the positrons (1c and 1d) of the thermal bath. Through its interactions with the spectator particles, this current couples to the electromagnetic field $A^{\beta}$, hence an effective electric charge (or charge radius) for the neutrinos which propagate inside a plasma.

Fig. 2 One-loop Feynman diagram contribution to the electromagnetic interactions of neutrinos, in finite temperature field theory.

Fig. 3 The total neutrino emissivity $\epsilon$ as a function of the plasma density $\rho$ for pure helium, at $T=10^{8} \mathrm{~K}$. Fig. 3a shows the exact absolute rate whilst fig. $3 \mathrm{~b}$ presents the ratios between various approximations and the exact rate.

Fig. 4 The neutrino emissivity as a function of the plasma temperature for pure carbon, at $\rho=1.8 \times 10^{6} \mathrm{~g} / \mathrm{cm}^{3}$. Fig. 4 a shows the exact absolute rate whilst fig. $4 \mathrm{~b}$ exhibits the ratios between various approximations and the exact rate. 